\title{
https://doi.org/10.1007/s10357-021-3849-8 \\ Die Regulierung der Einführung invasiver gebietsfremder Arten durch Biofouling an der Schnittstelle zwischen Naturschutzrecht und Seeschifffahrtsrecht
}

\author{
Valentin J. Schatz
}

() Der/die Autor(en) 2021. Dieser Artikel ist eine Open-Access-Publikation.

\begin{abstract}
Bewuchs an Seeschiffen (sog. Biofouling) ist ein bedeutender Vektor bei der Einführung invasiver gebietsfremder aquatischer Arten und damit eine Herausforderung für den Naturschutz. Da es sich hierbei - wie beim bereits speziell regulierten Ballastwasser - um ein systemisches Problem der Seeschifffahrt handelt, bedarf es eines speziellen Regelungssystems an der Schnittstelle zwischen Naturschutzrecht (BNatSchG) und Seeschifffahrtsrecht (SeeAufgG und SeeUmwVerhV). Vor diesem Hintergrund untersucht dieser Beitrag die rechtliche Regulierung von Biofouling Management. Er geht zunächst auf den völkerrechtlichen Rahmen und die Guidelines der Internationalen Seeschifffahrtsorganisation (IMO) ein. Danach wendet sich der Beitrag den einschlägigen Instrumenten des Unionsrechts zu. Zuletzt untersucht er das nationale Recht, wobei im Lichte der bisherigen Regulierung in Neuseeland, Kalifornien und Australien auf künftige gesetzgeberische Optionen in Deutschland eingegangen wird.
\end{abstract}

\section{Einleitung}

Im marinen Sektor versteht man unter Biofouling (bzw. schlicht Fouling) den Bewuchs bzw. die Besiedelung von unter Wasser befindlichen Oberflächen, wie beispielsweise Schiffsrümpfen oder Offshore-Anlagen, durch Mikroorganismen (z. B. Bakterien, einzellige Algen, Tiere und Pilze) und Makroorganismen (z. B. Muscheln, Seepocken und Algen). ${ }^{1}$ Biofouling beginnt regelmäßig damit, dass zunächst Mikroorganismen bzw. von ihnen ausgeschiedene Stoffe einen schleimigen Biofilm auf einer Oberfläche bilden, der in der Folge von Makroorganismen bzw. deren Larven und Sporen besiedelt wird. Sowohl die Besiedelung als auch deren Ausbreitung erfolgt - u. a. abhängig von physikalischen und biologischen Umständen - oft sehr schnell. ${ }^{2}$

Dieser Bewuchs bringt eine Reihe von ökologischen und ökonomischen Problemen mit sich. Insbesondere kann Biofouling ein Vektor beim Transport von invasiven gebietsfremden Arten in aquatische Okosysteme sein. ${ }^{3}$ Invasive gebietsfremde Arten können wiederum nicht nur solche Okosysteme schädigen (etwa durch die Verdrängung heimischer Arten), sondern dadurch auch weitergehende, insbesondere ökologische und ökonomische Schäden verursachen. ${ }^{4} \mathrm{Um}$ diese Risiken von Biofouling zu minimieren, können Schiffsbetreiber verschiedene Prozeduren, Kontrollen und Maßnahmen durchführen, die unter dem Überbegriff Biofouling Management zusammengefasst werden. ${ }^{5}$ Dazu können beispielsweise das Aufstellen von auf ein bestimmtes Seeschiff zugeschnittenen Biofouling Management Plänen, die Behandlung von Schiffsrümpfen mit geeigneten Antifouling-Beschichtungen (AFS) ${ }^{6}$, regelmäßige Verfahren zur Reinigung von Schiffsrümpfen ${ }^{7}$, In-

Valentin J. Schatz, Referendar am Hanseatischen Oberlandesgericht, Wissenschaftlicher Mitarbeiter

am Institut für Seerecht und Seehandelsrecht, Universität Hamburg, Hamburg, Deutschland spektionen und die Dokumentation solcher Maßnahmen gehören.

Vor diesem Hintergrund untersucht dieser Beitrag die rechtliche Regulierung von Biofouling Management. Der Fokus des Beitrags liegt dabei auf der Regulierung durch Hafenstaaten, ${ }^{8}$ sowie auf der Problematik der Einführung invasiver gebietsfremder Arten. ${ }^{9}$ Er geht zunächst auf den

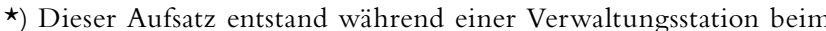
Bundesamt für Seeschifffahrt und Hydropgraphie (BSH). Er gibt allein die Auffassung des Autors wieder. Besonderer Dank für wertvolle Anmerkungen gilt Professor Dr. Dr. h.c. Peter Ehlers (Präsident des BSH a.D.), Dr. Susanne Heitmüller (BSH) und Hannelore Keim (Bundesministerium für Verkehr und digitale Infrastruktur (BMVI)). Dank für Unterstützung bei der Vorbereitung des Manuskripts gilt zudem Emma Bartmann, Christopher Janz und Torben Wassermann, jeweils studentische Hilfskräfte am Lehrstuhl für Internationales Seerecht und Umweltrecht, Völkerrecht und Öffentliches Recht (Professor Alexander Proelß) an der Universität Hamburg

1) Umweltbundesamt (UBA), Bewuchsatlas: Was ist Bewuchs?, Stand 19.4.2021, abrufbar unter https://www.umweltbundesamt.de/bewuchsatlas-was-ist-bewuchs; Bundesamt für Seeschifffahrt und Hydrographie (BSH), Biofouling, Stand 19.4.2021, abrufbar unter https://www.bsh.de/DE/THEMEN/Schifffahrt/ Umwelt_und_Schifffahrt/Biofouling/biofouling_node.html; jsessionid=255D5C629E3519C445D7F5F8EE8FB9C3.live11291.

2) Siehe dazu etwa Watermann et al., Gutachten zum regionalen Bewuchsdruck von Biofouling-Organismen in Deutschland, 2019.

3) Siehe allgemein International Maritime Organization (IMO), Biofouling, Stand 19.4.2021, abrufbar unter https://www.imo.org/en/ OurWork/Environment/Pages/Biofouling.aspx. Die IMO führt auch eine (nicht abschließende) Liste häufiger durch Biofouling an Schiffsrümpfen eingetragener invasiver gebietsfremder Organismen. Siehe IMO, Common Hull Fouling Invasive Species, Stand 19. 4.2021, abrufbar unter https://www.imo.org/en/OurWork/Environment/Pages/Common-Hull-Fouling-Invasive-Species.aspx.

4) Vgl. z. B. Hanley/Roberts, The Economic Benefits of Invasive Species Management, People and Nature 2019, 124

5) Vgl. etwa Davidson et al., Mini-Review: Assessing the Drivers of Ship Biofouling Management: Aligning Industry and Biosecurity Goals, Biofouling 2016, 411.

6) UBA, Antifouling-Mittel, Stand 19.4.2021, abrufbar unter https://www.umweltbundesamt.de/themen/chemikalien/biozide/biozidprodukte/antifouling-mittel.

7) Siehe etwa INTERTANKO, Intertanko Guide to Modern Antifouling Systems and Biofouling Management v. 2016, Stand 19.4.2021, abrufbar unter https://www.maritimecyprus.com/ 2016/02/17/intertanko-guide-to-modern-antifouling-systemsand-biofouling-management/.

8) Der Beitrag geht also nicht detailliert auf die Regulierungsmöglichkeiten von Flaggenstaaten ein.

9) Nicht im Detail behandelt werden daher beispielsweise Maßnahmen des Biofouling Managements, die primär der Herstellung und Aufrechterhaltung der Energieeffizienz von Seeschiffen dienen. Auch die wasser- und seeumweltrechtliche Zulässigkeit einzelner Maßnahmen des Biofouling Managements, wie etwa der Unterwasserreinigung von Schiffsrümpfen, wird nicht im Detail behandelt. 
völkerrechtlichen Rahmen und dort insbesondere auf die Regulierung von Biofouling Management durch die Internationale Seeschifffahrtsorganisation (IMO) ein. Danach wendet sich der Beitrag den einschlägigen Instrumenten des Unionsrechts zu. Zuletzt untersucht der Beitrag das nationale Recht (und dort vor allem das Verhältnis zwischen Naturschutzrecht und Seeschifffahrtsrecht), wobei insbesondere auf künftige Regulierungsoptionen eingegangen wird. Die Ergebnisse der Untersuchung sind in einer Schlussbetrachtung zusammengefasst.

\section{Völkerrechtliche Vorgaben}

Auf der Ebene des Völkerrechts sind vor allem das Seerechtsübereinkommen der Vereinten Nationen von 1982 (SRÜ) sowie von der IMO eingeführte Regelungen für den Umgang mit Biofouling relevant. Aus Platzgründen kann hier nicht auf die vielfältigen weiteren völkerrechtlichen Instrumente eingegangen werden, die die Einführung gebietsfremder invasiver Arten (mit) betreffen. ${ }^{10}$

\subsection{Seevölkerrechtliche Vorgaben (SRÜ)}

Teil XII des SRÜ enthält neben Regelungen zum Schutz der Meeresumwelt vor Meeresverschmutzung auch solche, die die Einführung invasiver gebietsfremder Arten betreffen. Bereits die allgemeine Verpflichtung des Art. 192 SRÜ, „,die Meeresumwelt zu schützen und zu bewahren“, erfasst mit ihrer holistischen Formulierung nicht nur die Meeresverschmutzung durch schädliche Stoffe, sondern auch den Schutz der natürlichen Meeresumwelt vor der Einführung invasiver gebietsfremder Arten. ${ }^{11}$

Die Gefahr, die von solchen Arten für die Meeresumwelt ausgeht, wird in Art. 196 Abs. 1 SRÜ aufgegriffen. Diese Vorschrift verpflichtet die Vertragsparteien zur Ergreifung, ,alle[r] notwendigen Maßnahmen zur Verhütung, Verringerung und Überwachung [...] der absichtlichen oder zufälligen Zuführung fremder oder neuer Arten in einen bestimmten Teil der Meeresumwelt, die dort beträchtliche und schädliche Veränderungen hervorrufen". Hier wiedergegeben sind nur die Teile der leider irreführenden (und nicht authentischen ${ }^{12}$ ) deutschen Übersetzung des SRÜ, die Art. 196 Abs. 1 SRÜ als eine anstatt von richtigerweise zwei separaten Verpflichtungen darstellt. ${ }^{13}$ Der authentische englische Wortlaut und die Historie der Norm sprechen sehr deutlich dafür, dass Art. 196 Abs. 1 Alt. 2 SRÜ eine separate Verpflichtung enthält, die auf die Erhaltung des natürlichen Zustands der Meeresumwelt abzielt und nicht lediglich auf eine Vermeidung von Meeresverschmutzungen. ${ }^{14}$

Anders als die deutsche Übersetzung von Art. 196 Abs. 1 SRÜ es impliziert, darf auch bezweifelt werden, dass die ,absichtliche oder zufällige Zuführung fremder oder neuer Arten in einen bestimmten Teil der Meeresumwelt" als „,Meeresverschmutzung“" i. S. d. Art. 1 Abs. 4 SRÜ zu klassifizieren ist, da sich die Einführung invasiver gebietsfremder Arten kaum unter diese Definition subsumieren lässt. ${ }^{15}$ Damit sind auch die allgemeinen, die Meeresverschmutzung betreffenden Vorschriften einschließlich Art. 194 SRU nicht ohne weiteres auf den Eintrag invasiver Arten anwendbar. ${ }^{16}$

Im Kontext von Biofouling sind vor allem ,fremde Arten“ i. S. d. Art. 196 Abs. 1 SRÜ von Bedeutung. Nach einer gängigen, aber nicht für das SRÜ verbindlichen Definition sind das solche Arten, Unterarten oder niedrigere Taxa, die außerhalb ihres früheren oder derzeitigen natürlichen Verbreitungsgebiets und Ausbreitungspotenzials (d.h. außerhalb des Gebiets, das sie natürlich besetzen oder das sie ohne direkte oder indirekte Einführung oder Pflege durch den Menschen nicht besetzen könnten) vorkommen, und schließt alle Teile, Gameten oder Fortpflanzungsorgane solcher Arten ein, die überleben und sich an- schließend fortpflanzen könnten. ${ }^{17}$ Unter Rückgriff auf Art. 8 lit. h des Übereinkommens über die biologische Vielfalt von 1993 (CBD), der eine Art. 196 Abs. 1 Alt. 2 SRÜ ähnliche Verpflichtung enthält, ${ }^{18}$ können solche Arten als ,invasive fremde Arten“ angesehen werden, die Ökosysteme, Lebensräume oder heimische Arten gefährden. ${ }^{19}$ Der Wortlaut ,die dort beträchtliche und schädliche Veränderungen hervorrufen können“ dürfte so zu interpretieren sein, dass es gerade auch um potenzielle, noch nicht erwiesene Veränderungen geht, die einer frühen Beurteilung und ggf. Reaktion bedürfen, womit Art. 196 Abs. 1 Alt. 2 SRU dem Vorsorgeprinzip Rechnung trägt bzw. zu tragen vermag. ${ }^{20}$

Nach der ständigen Rechtsprechung des ITLOS und internationaler Schiedsgerichte ist der Begriff der Meeresumwelt in Teil XII des SRÜ räumlich weit zu verstehen und umfasst daher alle marinen Ökosysteme in allen maritimen Zonen, einschließlich der inneren Gewässer, des Küstenmeeres, der Archipelgewässer, der ausschließlichen Wirt-

10) Vgl. für einen Überblick Holljesiefken, Die rechtliche Regulierung invasiver gebietsfremder Arten in Deutschland: Bestandsaufnahme und Bewertung, 2007, S. $54 \mathrm{ff}$.

11) Czybulka, in: Proelss, United Nations Convention on the Law of the Sea (UNCLOS): A Commentary, 2017, Art. 192, Art. 196 Rdnr. 9.

12) Vgl. Art. 320 SRÜ.

13) Zink, Das Ballastwasser-Übereinkommen der internationalen Seeschifffahrts-Organisation von 2004: Anwendung in Nordund Ostsee, 2016, S. 123 ff.; Proelß/Schatz, Rechtliche Vorgaben zum Umgang mit Schiffsabwasser: Völker-, unions- und nationalrechtliche Anforderungen an Einleitungen von ScrubberAbwasser, Ballastwasser und häuslichem Abwasser durch Schiffe, 2019, S. 85.

14) Nordquist/Grandy/Rosenne/Yankov, United Nations Convention on the Law of the Sea 1982: A Commentary (Vol. 4), 1991, S. $73 \mathrm{ff}$; Zink, Das Ballastwasser-Übereinkommen der internationalen Seeschifffahrts-Organisation von 2004: Anwendung in Nord- und Ostsee, 2016, S. 123 ff.; Czybulka, in: Proelss, United Nations Convention on the Law of the Sea (UNCLOS): A Commentary, 2017, Art. 196 Rdnr. 1.

15) Czybulka, in: Proelss, United Nations Convention on the Law of the Sea (UNCLOS): A Commentary, 2017, Art. 196 Rdnr. 20; Differenzierend Zink, Das Ballastwasser-Übereinkommen der internationalen Seeschifffahrts-Organisation von 2004: Anwendung in Nord- und Ostsee, 2016, S. 128 ff., der darauf hinweist, dass invasive gebietsfremde Arten in Stoffen (z. B. Wasser oder Biomasse) enthalten sein können, die ihrerseits als „Meeresverschmutzung" i.S.d. Art. 1 Abs. 4 SRÜ zu qualifizieren sind.

16) Siehe aber die teilweise geäußerten Einwände in der vorhergehenden Fußnote.

17) Frei übersetzt nach: IUCN, Guidelines for the Prevention of Biodiversity Loss Caused by Alien Invasive Species v. 2000, Stand 19.4.2021, abrufbar unter http://www.issg.org/pdf/guidelines_ iucn.pdf, Rdnr. 3; siehe Czybulka, in: Proelss, United Nations Convention on the Law of the Sea (UNCLOS): A Commentary, 2017, Art. 196 Rdnr. 14.

18) Vgl. dazu Holljesiefken, Die rechtliche Regulierung invasiver gebietsfremder Arten in Deutschland: Bestandsaufnahme und Bewertung, 2007, S. 54 ff., S. 59 ff.; Zink, Das Ballastwasser-Übereinkommen der internationalen Seeschifffahrts-Organisation von 2004: Anwendung in Nord- und Ostsee, 2016, S. $57 \mathrm{ff}$.

19) Czybulka, in: Proelss, United Nations Convention on the Law of the Sea (UNCLOS): A Commentary, 2017, Art. 196 Rdnr. 14; Proelß/Schatz, Rechtliche Vorgaben zum Umgang mit Schiffsabwasser: Völker-, unions- und nationalrechtliche Anforderungen an Einleitungen von Scrubber-Abwasser, Ballastwasser und häuslichem Abwasser durch Schiffe, 2019, S. 85. Definitionen aus der CBD wurden auch bereits von der Rechtsprechung zur Auslegung nicht definierter Begriffe des SRÜ herangezogen, was im Rahmen der allgemeinen Vertragsauslegungsregeln auch zulässig ist. Vgl. PCA, South China Sea Arbitration (Republic of the Philippines v. People's Republic of China), Award v. 12.6.2016, PCA Case No. 2013-19, Rdnr. 945.

20) Czybulka, in: Proelss, United Nations Convention on the Law of the Sea (UNCLOS): A Commentary, 2017, Art. 196 Rdnr. 14. 
schaftszone (AWZ), des Festlandsockels, des Tiefseebodens und der hohen See..$^{21}$ Auch die Verpflichtung aus Art. 196 Abs. 1 Alt. 2 SRÜ ist damit in Meeresgewässern mindestens bis zur Süßwassergrenze anwendbar. ${ }^{22}$ Bei einem weiten, funktionalen Verständnis der inneren Gewässer, wie es häufig auch dem umweltbezogenen Seeschifffahrtsrecht zugrunde liegt, dürften auch Seeschifffahrtsstraßen und Reeden sowie an Binnenwasserstraßen gelegene Seehäfen erfasst sein. ${ }^{23}$

Art. 196 Abs. 1 Alt. 2 SRÜ ist als Sorgfaltspflicht ausgestaltet (,,shall take all measures necessary to prevent, reduce and control"). Erfüllt wird diese Verpflichtung durch die Ergreifung der Maßnahmen (insbesondere gesetzliche Regelungen und deren Durchsetzung), die notwendig sind, um beträchtliche und schädliche Auswirkungen auf die Meeresumwelt $\mathrm{zu}$ verhüten, zu verringern und $\mathrm{zu}$ überwachen, die durch den Eintrag von fremden oder neuen Organismen entstehen. Die Hauptverantwortung liegt bei den Flaggenstaaten, die für Schiffe unter ihrer Flagge verbindliche Standards einführen und durchsetzen können. ${ }^{24}$ Ergänzend - oder daneben - können aber auch Hafenstaaten durch (unilaterale) Maßnahmen den Zugang zu ihren Häfen an Umweltstandards knüpfen, die dem Schutz ihrer (Meeres-) Gewässer und Biodiversität dienen. ${ }^{25}$ Auf globaler Ebene besteht gemäß Art. 196 Abs. 1 Alt. 2 i. V.m. Art. 197 SRÜ die Verpflichtung, bei der internationalen Regulierung und Ausarbeitung von Empfehlungen zu kooperieren, ${ }^{26}$ was - wie unten aufgezeigt - im Hinblick auf Biofouling zumindest in Ansätzen auch bereits im Rahmen der IMO geschehen ist.

\subsection{IMO Biofouling-Richtlinien}

Die IMO ist für die Regulierung der Einführung von invasiven gebietsfremden Arten durch die Seeschifffahrt von zentraler Bedeutung. ${ }^{27}$ Bisher hat sie keine verbindlichen globalen Regelungen zum Biofouling Management erarbeitet, die einheitlich von den Flaggenstaaten umzusetzen sind, weshalb bisher vor allem unilaterale Regulierung einzelner Hafenstaaten zu beobachten ist (siehe dazu noch unten). Insbesondere behandelt das Übereinkommen über Bewuchsschutzsysteme von 2001 (AFS-Übereinkommen) lediglich die schädlichen Umweltauswirkungen durch die Inhaltsstoffe bestimmter in AFS verwendeter Biozide. Auch das Internationale Übereinkommen zur Kontrolle und Behandlung von Ballastwasser und Sedimenten von Schiffen von 2004 (BWÜ) behandelt lediglich die Problematik der Einführung invasiver gebietsfremder Arten durch Ballastwasser, nicht aber durch Biofouling. ${ }^{28}$

Über diese beiden Instrumente hinaus hat der Ausschuss für den Schutz der Meeresumwelt (Marine Environment Protection Committee, MEPC) internationale Maßnahmen zur Vermeidung und Verringerung der Verbreitung invasiver Arten durch Biofouling am Schiffsrumpf intensiv beraten und in 2011 die IMO Biofouling-Richtlinien (MEPC.207(62)) verabschiedet. ${ }^{29}$ Die Richtlinien enthalten praktische Empfehlungen für Maßnahmen, die dazu beitragen sollen, die Risiken der Verbreitung von aquatischen invasiven gebietsfremden Arten durch Biofouling sowie ökologische Risiken von Biofouling Management zu minimieren. ${ }^{30}$ Sie wurden 2012 durch einen Leitfaden für Besitzer und Betreiber von Sportbooten ergänzt. ${ }^{31}$

Die Biofouling-Richtlinien sollen Empfehlungen für allgemeine Maßnahmen zur Minimierung der mit Biofouling verbundenen Risiken für alle Arten von Schiffen geben und richten sich an staatliche und zivile Akteure. ${ }^{32}$ Zur Zielerreichung wird ein Instrument des schiffsspezifischen Biofouling Managements entwickelt. ${ }^{33}$

$\mathrm{Zu}$ den in den Biofouling-Richtlinien vorgeschlagenen Maßnahmen des Biofouling Managements gehören insbesondere ein Biofouling Management Plan und ein Biofouling Record Book. Im Biofouling Management Plan sind die einzelnen von einem Schiff vorzunehmenden Prozeduren, Kontrollen und Maßnahmen aufgelistet. ${ }^{34} \mathrm{Im}$ Biofouling Record Book werden die bisherigen Maßnahmen dokumentiert. ${ }^{35}$ Des Weiteren enthalten die BiofoulingRichtlinien detaillierte Empfehlungen zur gezielten Auswahl geeigneter AFS Beschichtungssysteme sowie deren Kontrolle und ggf. Pflege, die ausdrücklich als das wichtigste Mittel zur Verhinderung und Bekämpfung von Biofouling auf den unter Wasser befindlichen Oberflächen von Schiffen bezeichnet werden. ${ }^{36}$ Daneben werden Empfehlungen zur Unterwasserinspektion, -reinigung und -wartung gegeben. ${ }^{37}$

Bei neuen Schiffen stufen die Biofouling-Richtlinien den Schiffsentwurf und -bau als das umfassendste, wirksamste und dauerhafteste Mittel zur Minimierung der Biofouling-Risiken ein und geben auch hierzu Empfehlungen. ${ }^{38}$ Staaten sollen zudem Informationen, die für die

21) Vgl. ITLOS, Request for an Advisory Opinion Submitted by the Sub-Regional Fisheries Commission (SRFC), Advisory Opinion v. 2.4.2015, ITLOS Rep (2015) 4, Rdnr. 120; PCA, Dispute Concerning Coastal State Rights in the Black Sea, Sea of Azov, and Kerch Strait (Ukraine v. the Russian Federation), Award concerning the Preliminary Objections of the Russian Federation v. 21.2.2020, PCA Case No. 2017-06, Rdnr. 295

22) Vgl. Graf Vitzthum, in: FS für Tomschuat, 2006, S. 1067, $1070 \mathrm{ff}$

23) Siehe zu einem solchen funktionalen Verständnis Graf Vitzthum, in: ders., Handbuch des Seerechts, 2006, S. 63, 84-85. Vgl. im Kontext des MARPOL Übereinkommens auch Proelß/Schatz, Rechtliche Vorgaben zum Umgang mit Schiffsabwasser: Völker-, unions- und nationalrechtliche Anforderungen an Einleitungen von Scrubber-Abwasser, Ballastwasser und häuslichem Abwasser durch Schiffe, 2019, S. 42

24) Siehe etwa Harrison, Saving the Oceans Through Law: The International Legal Framework for the Protection of the Marine Environment, 2017, 139-145.

25) Siehe zu diesem Themenkomplex neuerdings Coelho, Unilateral Port State Jurisdiction: The Quest for Universality in the Prevention, Reduction and Control of Ship-Source Pollution, 2019; Honniball, Extraterritorial Port State Measures: The Basis and Limits of Unilateral Port State Jurisdiction to Combat Illegal, Unreported and Unregulated Fishing, 2019.

26) Zum Teil wird auch eine Anwendung von Art. 211 SRÜ auf die Einführung invasiver gebietsfremder Arten (etwa durch Ballastwasser) durch Schiffe als möglich erachtet, obwohl diese Vorschrift nach ihrem Wortlaut nur auf „Meeresverschmutzung“ i.S.d. Art. 1 Abs. 4 SRÜ anwendbar ist. Vgl. Zink, Das Ballastwasser-Übereinkommen der internationalen SeeschifffahrtsOrganisation von 2004: Anwendung in Nord- und Ostsee, 2016, S. $154 \mathrm{ff}$.; Bartenstein, in: Proelss, United Nations Convention on the Law of the Sea (UNCLOS): A Commentary, 2017, Art. 211 Rdnr. 2.

27) Vgl. beispielsweise United Nations (UN), The Future We Want: Outcome Document of the United Nations Conference on Sustainable Development v. 22.6.2012, Stand 19.4.2021, abrufbar unter https://sustainabledevelopment.un.org/content/documents/733FutureWeWant.pdf, Rdnr. 164.

28) Siehe zum BWÜ allgemein Zink, Ocean Yearbook 2015, 441; Zink, Das Ballastwasser-Übereinkommen der internationalen Seeschifffahrts-Organisation von 2004: Anwendung in Nordund Ostsee, 2016, $123 \mathrm{ff}$.

29) IMO, Guidelines for the Control and Management of Ships' Biofouling to Minimize the Transfer of Invasive Aquatic Species v. 15.7.2011, Res. MEPC.207(62)

30) Vgl. Ziff. 4.1 Biofouling-Richtlinien.

31) IMO, Guidance for Minimizing the Transfer of Invasive Aquatic Species as Biofouling (Hull Fouling) for Recreational Craft v. 12.11.2012, MEPC.1/Circ.792; vgl. auch Ziff. 3.2 BiofoulingRichtlinien.

32) Ziff. 3.1 S. 1 Biofouling-Richtlinien.

33) Ziff. 4.2 S. 1 Biofouling-Richtlinien.

34) Vgl. Ziff. 5.2-5.4 sowie Anhang 1 Biofouling-Richtlinien.

35) Vgl. Ziff. 5.5-5.8 sowie Anhang 2 Biofouling-Richtlinien.

36) Vgl. Ziff. 6.1-6.9 Biofouling-Richtlinien.

37) Vgl. Ziff. 7.1-7.13 Biofouling-Richtlinien.

38) Vgl. Ziff. 8.1-8.3 Biofouling-Richtlinien. 
Biofouling-Richtlinien von Belang sind, sammeln und der Organisation zum Informationsaustausch zur Verfügung stellen. ${ }^{39}$ Ein weiterer von den Biofouling-Richtlinien hervorgehobener Aspekt, ist die fachgerechte Ausbildung und Schulung von Schiffskapitänen und -besatzungen, Betreiberinnen von Reinigungs- oder Wartungsanlagen im Wasser und gegebenenfalls von Besichtigern oder Inspektorinnen von Schiffen auf der Grundlage der in den Biofouling-Richtlinien enthaltenen Informationen. ${ }^{40}$ Abschließend finden sich in Ziff. 11 der Biofouling-Richtlinien noch ergänzende Empfehlungen. Unter der Überschrift „Future Work“ werden noch Hinweise zur Notwendigkeit wissenschaftlicher Forschung im Hinblick auf die Entwicklung von Biofouling Management Technologien und die Beschaffung von Informationen über AFS und andere bereits existierende Maßnahmen aufgeführt. ${ }^{41}$

Die Staaten sollen selbst bestimmen, ob und inwieweit sie die Biofouling-Richtlinien in ihrer Jurisdiktion zur Anwendung bringen. ${ }^{42} \mathrm{Da}$ die Biofouling-Richtlinien somit nur Empfehlungen darstellen und nicht rechtlich verbindlich sind, können aus ihnen unmittelbar keine Vorgaben für das Biofouling Management hergeleitet werden. Ihnen kommt aber bisher nicht der Rang von ,allgemein anerkannten internationalen Regeln und Normen“i.S.d. Art. 211 Abs. 2 SRÜ zu, da sie nicht hinreichend konkret und preskriptiv konzipiert sind, um ihnen eindeutige Handlungsanweisungen zu entnehmen, was auch daraus ersichtlich wird, dass sie noch nicht von sehr vielen Staaten in ihrer nationalen Rechtsordnung für verbindlich erklärt wurden. ${ }^{43}$ Sie können unmittelbar daher nur Anhaltspunkte für Kriterien bieten, die Akteurinnen in der Industrie oder nationale Behörden bzw. den Gesetzgeber bei ihren Entscheidungen leiten können. Insbesondere könnte der Gesetzgeber die Biofouling-Richtlinien bzw. die darin enthaltenen Standards und Instrumente in von ihnen selbst weiter konkretisierte, verbindliche gesetzliche Bestimmungen gießen. $\mathrm{Zu}$ beachten ist allerdings, dass die Biofouling-Richtlinien derzeit einen Bewertungsund Prüfungsprozess innerhalb der IMO durchlaufen, für den 2013 ein spezieller Evaluationsleitfaden vorbereitet wurde. ${ }^{44}$

\subsection{Regionale Initiativen im Rahmen von HELCOM und OSPAR}

Die IMO Biofouling-Richtlinien wurden mittlerweile auch in regionalen Foren aufgegriffen. Für Deutschland bedeutsam ist zum einen die Baltic Marine Environment Protection Commission (Helsinki Commission, HELCOM), die auf Grundlage des Übereinkommens zum Schutz der Meeresumwelt des Ostseegebietes von 1992 (HELCOM Übereinkommen) ${ }^{45}$ errichtet wurde. Zum anderen ist im Hinblick auf die Nordsee das Übereinkommen zum Schutz der Meeresumwelt des Nordostatlantiks von 1992 (OSPAR Übereinkommen) ${ }^{46}$ von Bedeutung.

Im Rahmen von HELCOM und OSPAR gibt es bereits Bestrebungen hinsichtlich einer regional harmonisierenden Umsetzung der IMO Biofouling-Richtlinien, die insbesondere auf eine Initiative in der HELCOM/OSPAR Joint Task Group Ballast (JTG Ballast) zurückgehen. ${ }^{47}$ Unter anderem das EU INTERREG Baltic Sea Region Projekt mit dem Titel „Completing Management Options in the Baltic Sea Region to Reduce Risk of Invasive Species Introduction by Shipping" (COMPLETE), an dem Mitgliedstaaten von HELCOM und das HELCOM Sekretariat beteiligt sind, befasst sich im Kontext der Ostsee mit dem Thema Biofouling. ${ }^{48}$ In diesem Rahmen wurde ein Vorschlag für eine Regionale Biofouling Management Roadmap entwickelt. ${ }^{49}$ Bei der Roadmap handelt es sich um eine detaillierte Zusammenstellung von Informationen und Ausarbeitung von Empfehlungen im Hinblick auf die
Umsetzung von Biofouling Management in der Ostsee unter besonderer Berücksichtigung der IMO BiofoulingRichtlinien. ${ }^{50}$ Die Roadmap behandelt daher auch Themen wie den Biofouling Management Plan und das Biofouling Record Book ${ }^{51}$, die Unterwasserreinigung ${ }^{52}$ und die Verbreitung von Informationen. ${ }^{53}$

Die Roadmap wurde auf der 20. Sitzung der HELCOM Maritime Working Group im Oktober 2020 vorgestellt,

39) Vgl. Ziff. 9.1-9.4 Biofouling-Richtlinien.

40) Vgl. Ziff. 10.1-10.2 Biofouling-Richtlinien.

41) Vgl. Ziff. 12.1-12.3 Biofouling-Richtlinien.

42) Ziff. 3.1 S. 2 Biofouling-Richtlinien.

43) Vgl. dazu Bartenstein, in: Proelss, United Nations Convention on the Law of the Sea (UNCLOS): A Commentary, 2017, Art. 211 Rdnr. $12 \mathrm{ff}$.

44) IMO, Guidance for Evaluating the 2011 Guidelines for the Control and Management of Ships' Biofouling to Minimize the Transfer of Invasive Aquatic Species v. 13.6.2013, MEPC.1/ Circ.811.

45) BGBl. 1982 II S. 2; BGBl. 1996 II S. 399.

46) BGBl. 1994 II S. 1360.

47) Vgl. HELCOM/OSPAR JTG Ballast, OSPAR-EIHA Proposal for the Provisional Inclusion of Hull Fouling Issues to the ToR of the HELCOM/OSPAR TG BALLAST v. 29.11.2018, Stand 19.4.2021, abrufbar unter https://portal.helcom.fi/meetings/HELCOM-OSPAR\%20TG\%20BALLAST\%209-2018556/MeetingDocuments/7-1\%20OSPAR_EIHA\%20proposal $\% 20$ for $\% 20$ the $\% 20$ provisional $\% 20$ inclusion $\% 20$ of $\% 20$ hull $\%$ 20 fouling $\% 20$ issues $\% 20$ to $\% 20$ the $\% 20$ ToR $\% 20$ of $\% 20$ the $\%$ 20TG\%20BALLAST.pdf; HELCOM/OSPAR JTG Ballast, Outcome of the Ninth Meeting of the Joint HELCOM/OSPAR Task Group on Ballast Water Management Convention Exemptions v. 14.12.2018, Stand 19.4.2021, abrufbar unter https://portal.helcom.fi/meetings/HELCOM-OSPAR\%20TG\%20BALLAST\%209-2018-556/MeetingDocuments/Outcome\%20of\% 20TG\%20BALLAST\%209-2018.pdf, Ziff. 3.4-3.7, 7.1-7.8; HELCOM/OSPAR JTG Ballast, Outcome of the Tenth Meeting of the Joint HELCOM/OSPAR Task Group on Ballast Water Management Convention Exemptions v. 18.12.2019, Stand 19.4.2021, abrufbar unter https://portal.helcom.fi/meetings/ TG\%20BALLAST\%2010-2019-614/MeetingDocuments/Outcome $\% 20$ of $\% 20$ TG $\% 20$ BALLAST\%2010-2019.pdf, Ziff. 2.32.6, 7.1-7.6, 8.2-8.3, 9.3.

48) HELCOM, COMPLETE: Completing Management Options in the Baltic Sea Region to Reduce Risk of Invasive Species Introduction by Shipping, Stand 19.4.2021, abrufbar unter https:// helcom.fi/helcom-at-work/projects/complete/.

49) HELCOM, Proposal for a Regional Baltic Biofouling Management Roadmap v. 11.9.2020, Stand 19.4.2021, abrufbar unter https://portal.helcom.fi/meetings/MARITIME\%2020-2020787/MeetingDocuments/4-1\%20Proposal\%20for\%20a\%20Regional\%20Baltic\%20Biofouling\%20Management $\% 20$ Roadmap.pdf.

50) HELCOM, Proposal for a Regional Baltic Biofouling Management Roadmap v. 11.9.2020, Stand 19.4.2021, abrufbar unter https://portal.helcom.fi/meetings/MARITIME\%2020-2020787/MeetingDocuments/4-1\%20Proposal\%20for\%20a \%20Regional\%20Baltic\%20Biofouling\%20Management\%20Roadmap. pdf, S. $2 \mathrm{ff}$.

51) HELCOM, Proposal for a Regional Baltic Biofouling Management Roadmap v. 11.9.2020, Stand 19.4.2021, abrufbar unter https://portal.helcom.fi/meetings/MARITIME\%2020-2020787/MeetingDocuments/4-1\%20Proposal\%20for $\% 20 \mathrm{a} \% 20 \mathrm{Re}-$ gional $\% 20$ Baltic $\% 20$ Biofouling\%20Management\%20Roadmap. pdf, S. $3 \mathrm{ff}$.

52) HELCOM, Proposal for a Regional Baltic Biofouling Management Roadmap v. 11.9.2020, Stand 19.4.2021, abrufbar unter https://portal.helcom.fi/meetings/MARITIME\%2020-2020787/MeetingDocuments/4-1\%20Proposal\%20for\%20a\%20Regional\%20Baltic\%20Biofouling\%20Management\%20Roadmap. pdf, S. $6 \mathrm{ff}$.

53) HELCOM, Proposal for a Regional Baltic Biofouling Management Roadmap v. 11.9.2020, Stand 19.4.2021, abrufbar unter https://portal.helcom.fi/meetings/MARITIME\%2020-2020787/MeetingDocuments/4-1\%20Proposal\%20for\%20a\%20Regional $\% 20$ Baltic\%20Biofouling\%20Management $\% 20$ Roadmap. pdf, S. 13. 
ausdrücklich unterstützt und der JTG Ballast vorgelegt. ${ }^{54}$ Die JTG Ballast einigte sich zwar darauf, dass die Roadmap ein bedeutender Beitrag zur aktuell im Rahmen der IMO stattfindenden Arbeit sei, dass sie aber derzeit nicht als offizielles Instrument von HELCOM angenommen werden sollte; stattdessen solle die Roadmap unter Beachtung der aktuellen Entwicklungen auf Ebene der IMO der 59. Sitzung der Heads of Delegation zunächst nur zur Kenntnisnahme vorgelegt werden. ${ }^{55}$ Die 59. Sitzung der Heads of Delegation nahm dies zur Kenntnis und lud das zuständige Baltic Sea Action Plan (BSAP) Segment Team dazu ein, diese Einschätzung bei der Prüfung neuer Vorschläge für BSAP Maßnahmen zu berücksichtigen und auf die Roadmap zu verweisen. ${ }^{56}$ Daher dürfte derzeit nicht damit $\mathrm{zu}$ rechnen sein, dass die Roadmap bei HELCOM 42-2021 offiziell als HELCOM Instrument beschlossen wird.

\section{Unionsrechtliche Vorgaben}

Obwohl das Unionsrecht die Einführung gebietsfremder invasiver Arten ${ }^{57}$ in vielerlei Hinsicht thematisiert, ist Biofouling Management von Seeschiffen (abgesehen von der hier nicht behandelten Thematik der $\mathrm{AFS}^{58}$ ) bisher nicht Gegenstand spezieller unionsrechtlicher Regelungen. Allerdings wird die Thematik in der Verordnung über die Prävention und das Management der Einbringung und Ausbreitung invasiver gebietsfremder Arten (IAVO) ${ }^{59}$ und in Richtlinie (EG) 2008/56 zur Schaffung eines Ordnungsrahmens für Maßnahmen der Gemeinschaft im Bereich der Meeresumwelt (MSRL) ${ }^{60}$ aufgegriffen.

\subsection{Invasive-Arten-VO (IAVO)}

Die IAVO enthält eine Reihe von Regelungen, die für die Einführung von Biofouling-Organismen in das Meer relevant sind. Die Thematik Biofouling wird ausdrücklich allerdings nur in Erwägungsgrund 21 erwähnt. Dort heißt es, dass die nach der IAVO ergriffenen freiwilligen und verbindlichen Maßnahmen beispielsweise auch die in den IMO Biofouling-Richtlinien vorgeschlagenen Maßnahmen umfassen sollen. Damit ist jedoch keine Rechtspflicht der Mitgliedstaaten zur Umsetzung der IMO BiofoulingRichtlinien verbunden.

$\mathrm{Zu}$ den für Biofouling relevanten Regelungen zählt insbesondere die in Art. 7 Abs. 2 IAVO niedergelegte Pflicht der Mitgliedstaaten, alle notwendigen Schritte zu unternehmen, um die nicht vorsätzliche oder grob fahrlässige Einbringung oder Ausbreitung ,invasiver gebietsfremder Arten von unionsweiter Bedeutung “ zu verhindern. Daneben ist auch das Verbot in Art. 7 lit. h IAVO, vorsätzlich „,invasive gebietsfremde Arten von unionsweiter Bedeutung" in die Umwelt freizusetzen, unter Umständen relevant. Dafür müssten jeweils zunächst aber bekannte marine Organismen, welche für die Verbreitung durch Biofouling relevant sind, auf die Liste der, ,invasiven gebietsfremden Arten von unionsweiter Bedeutung" gem. Art. 4 IAVO gesetzt werden. ${ }^{61}$ Dies ist - soweit ersichtlich - bisher mit Ausnahme etwa der zum Teil auch in Bewuchs vorhandenen chinesischen Wollhandkrabbe (Eriocheir sinensis) oder der Amurgrundel (Perccottus glenii) nicht in großem Umfang geschehen. Hierbei handelt es sich um Arten, die im Süßwasser leben und derzeit von Schiffen insbesondere innerhalb einer Region noch weiterverbreitet werden. Daneben können die Mitgliedstaaten gem. \$12 Abs. 1 IAVO eine nationale Liste gebietsfremder Arten von Bedeutung für den jeweiligen Mitgliedstaat erstellen.

Ferner ist noch Art. 13 Abs. 2 IAVO zu nennen, der die Mitgliedstaaten zur Aufstellung eines Aktionsplans oder eines Pakets von Aktionsplänen für nach Art. 13 Abs. 1 IAVO ermittelten prioritären Pfade der nicht vorsätzlichen Einbringung und Ausbreitung invasiver gebietsfremder Arten von unionsweiter Bedeutung verpflichtet. Eine entspre- chende Pflicht des Bundesministeriums für Umwelt, $\mathrm{Na}$ turschutz und nukleare Sicherheit (BMUB) findet sich in $\$ 40 \mathrm{~d}$ Abs. $1 \mathrm{BNatSchG},{ }^{62}$ wobei diese nicht auf Arten in der Unionsliste beschränkt ist. ${ }^{63}$ Der aktuelle Entwurf des deutschen Aktionsplans enthält einen eigenen Abschnitt „Maßnahmenkatalog zum Pfad „Bewuchs/Anlagerung am Schiffsrumpf", dieser ist allerdings auf die weitere Verbreitung von Organismen durch die Binnenschifffahrt und dort insbesondere durch Biofouling an Sportbooten beschränkt. ${ }^{64}$

\subsection{Meeresstrategie-Rahmenrichtlinie (MSRL)}

Die MSRL dient gem. ihres Art. 1 Abs. 1 der Schaffung eines Rahmens, innerhalb dessen die Mitgliedstaaten die notwendigen Maßnahmen ergreifen, um spätestens bis zum Jahr 2020 einen guten Zustand der Meeresumwelt zu erreichen oder zu erhalten. Sie ist in allen „Meeresgewässern“ i.S.d. Art. 2 Abs. 1 MSRL anwendbar, wovon aus seevölkerrechtlicher Sicht Teile der inneren Gewässer, ${ }^{65}$ das Küs-

54) HELCOM/OSPAR JTG Ballast, Proposal for a Regional Baltic Biofouling Management Roadmap v. 27.11.2020, Stand 19.4.2021, abrufbar unter https://portal.helcom.fi/meetings/ TG\%20BALLAST\%2011-2020-763/MeetingDocuments/ 0702 Rev. $\% 201 \% 20$ Proposal $\% 20$ for $\% 20 \mathrm{a} \% 20$ Regional $\% 20 \mathrm{Bal}$ tic\%20Biofouling\%20Management\%20Roadmap.pdf.

55) HELCOM/OSPAR JTG Ballast, Outcome of the Eleventh Meeting of the Joint HELCOM/OSPAR Task Group on Ballast Water Management Convention (BWMC) and Biofouling v. 27.11.2020, Stand 19.4.2021, abrufbar unter: https://portal.helcom.fi/meetings/TG\%20BALLAST\%2011-2020-763/MeetingDocuments/Outcome\%20TG\%20BALLAST\%2011-2020.pdf, Ziff. 7.1-7.13.

56) HELCOM, Outcome of the 59th Meeting of the Heads of Delegation (HOD 59-2020) v. 8.12.2020, Stand 19.4.2021, abrufbar unter https://portal.helcom.fi/meetings/HOD\%20592020-784/MeetingDocuments/Outcome\%20of\%20HOD\% 2059-2020.pdf, Ziff. 6.22-6.23.

57) Vgl. die Übersicht bei Holljesiefken, Die rechtliche Regulierung invasiver gebietsfremder Arten in Deutschland: Bestandsaufnahme und Bewertung, 2007, S. $120 \mathrm{ff}$

58) Siehe Verordnung (EU) Nr. 528/2012 des Europäischen Parlaments und des Rates v. 22.5.2012 über die Bereitstellung auf dem Markt und die Verwendung von Biozidprodukten, ABl. 2012 L 167, S. 1, Produktart 21 (,Antifouling-Produkte“).

59) Verordnung (EU) Nr. 1143/2014 des Europäischen Parlaments und des Rates v. 22.10.2014 über die Prävention und das Management der Einbringung und Ausbreitung invasiver gebietsfremder Arten, ABl. 2014 L 317, S. 35; in Deutschland umgesetzt durch das Gesetz zur Durchführung der Verordnung (EU) Nr. 1143/2014 über Prävention und Management der Einbringung und Ausbreitung invasiver gebietsfremder Arten, BGBl. I, S. 3370 .

60) Richtlinie 2008/56/EG des Europäischen Parlaments und des Rates v. 17.6.2008 zur Schaffung eines Ordnungsrahmens für Maßnahmen der Gemeinschaft im Bereich der Meeresumwelt, ABl. 2008 L 164, S. 19.

61) Für die aktuelle Liste, siehe die Durchführungsverordnung (EU) 2019/1262 der Kommission v. 25.7.2019 zur Änderung der Durchführungsverordnung (EU) 2016/1141 zwecks Aktualisierung der Liste invasiver gebietsfremder Arten von EU-weiter Bedeutung, ABl. 2019 L 199, S. 1.

62) Gesetz über Naturschutz und Landschaftspflege (BNatSchG) v. 29. 7. 2009, BGB1. I S. 2542, zuletzt geändert durch Art. 290 der Verordnung v. 19. 6.2020, BGB1. I S. 1328.

63) Vgl. Gellermann, in: Landmann/Rohmer, Umweltrecht, 92. EL Februar 2020, BNatSchG $\$ 40 \mathrm{~d}$ Rdnr. $3 \mathrm{ff}$.

64) Aktionsplan gemäß Artikel 13 der Verordnung (EU) Nr. 1143/2014 des Europäischen Parlaments und des Rates v. 22.10.2014 über die Prävention und das Management der Einbringung und Ausbreitung invasiver gebietsfremder Arten (Entwurf) v. 13.8.2020, Stand 19.4.2021, abrufbar unter https:// www.bmu.de/fileadmin/Daten_BMU/Download_PDF/Artenschutz/entwurf aktionsplan_ias 2020 bf.pdf, S. 46-50

65) Vgl. Art. 2 Abs. 1 i. V.m. Art. 3 Nr. 1 lit. b MSRL. 
tenmeer, der Festlandsockel und die AWZ umfasst sind. ${ }^{66}$ Die MSRL will durch eine Anwendung des Ökosystemansatzes gewährleisten, dass die Gesamtbelastung der Meeresgewässer durch anthropogene Einwirkungen auf ein $\mathrm{Maß}$ beschränkt bleibt, das mit der Erreichung eines guten Umweltzustands vereinbar ist, damit die Meeresgewässer auf Dauer ihre Ökosystemleistungen erbringen können. ${ }^{67}$ Sie enthält selbst keine Regelungen, die unmittelbar der Erreichung eines guten Zustands der Meeresumwelt dienen. Stattdessen verpflichtet die MSRL die Mitgliedstaaten zur Entwicklung und Umsetzung von Meeresstrategien, die der Erreichung eines guten Zustands ,ihrer“ Meeresumwelt i.S.d. MSRL dienen. ${ }^{68}$ Diese Strategien sollen dazu dienen, ,die Meeresumwelt zu schützen und zu erhalten, ihre Verschlechterung zu verhindern oder, wo durchführbar, Meeresökosysteme in Gebieten, in denen sie geschädigt wurden, wiederherzustellen." ${ }^{69}$

Gemäß Art. 5 Abs. 2 MSRL gliedert sich das Verfahren zur Entwicklung von Meeresstrategien in sechs Schritte (sog. Aktionsplan), die wiederum zwei Phasen - die Vorbereitung und die Maßnahmenprogramme - zugeordnet sind. ${ }^{70}$ Die Vorgaben der MSRL wurden mit den $\$ S 45 \mathrm{a}$ ff. WHG in nationales Recht umgesetzt. ${ }^{71}$ Der auf Grundlage von $\$ 45 \mathrm{~h}$ Abs. $1 \mathrm{WHG}$ entwickelte Bericht des Bund/ Länder-Ausschusses Nord- und Ostsee (BLANO) zum MSRL-Maßnahmenprogramm über den Meeresschutz der deutschen Nord- und Ostsee vom 30.3.2016 ${ }^{72}$ spricht ausdrücklich davon, dass die ,durch menschliche Aktivitäten ausgelöste bzw. unterstützte Zuwanderung gebietsfremder Arten" durch Vorsorgemaßnahmen eingedämmt werden sollte. ${ }^{73}$ Diese Thematik ist dem Umweltziel UZ 3, mit dem Titel ,Meere ohne Beeinträchtigung der marinen Arten und Lebensräume durch die Auswirkungen menschlicher Aktivitäten“ zugeordnet. Dort werden als einschlägige Regelungsinstrumente unter anderem das BWÜ und die IAVO erwähnt, nicht aber die IMO Biofouling-Richtlinien. Allerdings geht beispielsweise der Bericht zum $\mathrm{Zu}-$ stand der deutschen Nordseegewässer aus dem Jahr 2018 ausdrücklich auf die IMO Biofouling-Richtlinien ein, welche ,zukünftig verstärkt betrachtet" werden sollen. ${ }^{74}$ Ein neuer Maßnahmenvorschlag mit dem Titel „Maßnahmen zur Umsetzung der IMO Biofouling Empfehlungen“" ist derzeit in der Entwicklung des kommenden Maßnahmenprogramm enthalten. Hier soll unter anderem die seitens des COMPLETE Projekts bei HELCOM vorgelegte Biofouling Roadmap beraten und der bereits vom Bundesamt für Seeschifffahrt und Hydrographie (BSH) etablierte „Runde Tisch Biofouling“" verstetigt werden. ${ }^{75}$

\section{Vorgaben im nationalen Recht}

Im deutschen Recht finden sich bisher keine speziell auf die Einführung invasiver gebietsfremder Arten durch Biofouling zugeschnittene Regelungen. Daher werden im Folgenden zunächst die naturschutzrechtlichen und seeschifffahrtsrechtlichen Rahmenbedingungen für eine künftige Regulierung des Biofouling Managements durch Deutschland als Hafenstaat dargelegt. Zuletzt wird ein rechtsvergleichender Blick auf die in Neuseeland, Kalifornien und Australien geschaffenen bzw. in der Schaffung befindlichen hafenstaatlichen Regelungen geworfen.

\subsection{Naturschutzrechtliche Vorgaben (BNatSchG)}

Die bereits erwähnte IAVO wurde in Deutschland in den of $40 \mathrm{a}-\mathrm{f}$ BNatSchG umgesetzt. ${ }^{76}$ Ermächtigungsgrundlagen für konkrete Maßnahmen finden sich in $\$ 40 \mathrm{a}$ BNatSchG.

4.1.1 Allgemeine Eingriffsbefugnisse des $\$ 40$ a Abs. 1 BNatSchG

Insbesondere ermächtigt $₫ 40 \mathrm{a}$ Abs. $1 \mathrm{BNatSchG}$ die zuständigen Naturschutzbehörden, nach pflichtgemäßem Ermes- sen „die im Einzelfall erforderlichen und verhältnismäßigen Maßnahmen " zu treffen. ${ }^{77}$ Diese Maßnahmen beziehen sich auf zwei verschiedene Tatbestände. Erstens gem. \40a Abs. 1 Nr. 1 BNatSchG auf Maßnahmen, die sicherstellen sollen, dass die Vorschriften der IAVO, des Kapitels 5 BNatSchG und der auf Grundlage der IAVO erlassenen Rechtsvorschriften in Bezug auf invasive Arten eingehalten werden. ${ }^{78}$ Zweitens auf Maßnahmen nach der eigenständigen Rechtsgrundlage des $\$ 40 \mathrm{a}$ Abs. 1 Nr. 2 BNatSchG, um die Einbringung oder Ausbreitung von invasiven Arten zu verhindern oder zu minimieren, ohne dass es dabei auf die Sicherstellung der Einhaltung von einschlägiger Vorschriften ankommt. ${ }^{79}$

4.1.2 Sonderregelung des $₫ 40$ a Abs. 6 BNatSchG für Maßnahmen zur Verhütung einer Verbreitung invasiver Arten durch Seeschiffe

Abweichend enthält $₫ 40$ a Abs. 6 BNatSchG eine Sonderregelung für die ,im Einzelfall erforderlichen Maßnahmen zur Verhütung einer Verbreitung invasiver Arten durch Seeschiffe" ${ }^{80}$ Nach dieser Vorschrift richten sich solche Maßnahmen nicht nach dem BNatSchG (als lex generalis), sondern nach dem SeeAufg $G^{81}$ und den auf Grundlage des SeeAufgG erlassenen Rechtsvorschriften (als lex specialis). ${ }^{82}$

66) Art. 2 Abs. 1 i. V.m. Art. 3 Nr. 1 lit. a MSRL. Dazu Proelß, in: Landmann/Rohmer Umweltrecht, 92. EL Februar 2020, WHG \43 Rdnr. 18 ff.; Proelß, in: Landmann/Rohmer, Umweltrecht, 92. EL Februar 2020, WHG $₫ 45$ a Rdnr. 17.

67) Vgl. im Einzelnen Art. 1 Abs. 3 MSRL.

68) Vgl. Art. 1 Abs. 2, 5 Abs. 1 MSRL.

69) Vgl. Art. 1 Abs. 2 lit. a MSRL.

70) Siehe im Detail Markus/Schlacke, ZUR 2009, 464, $468 \mathrm{f}$.

71) Vgl. Art. 1 des Gesetzes zur Umsetzung der MeeresstrategieRahmenrichtlinie sowie zur Änderung des BundeswasserstraBengesetzes und des Kreislaufwirtschafts- und Abfallgesetzes v. 6.10.2011, BGB1. I S. 1986.

72) Bund/Länder-Ausschuss Nord- und Ostsee: MSRL-Maßnahmenprogramm zum Meeresschutz der deutschen Nord- und Ostsee Bericht gemäß $₫ 45 \mathrm{~h}$ Absatz 1 des Wasserhaushaltsgesetzes, verabschiedet v. Bund/Länder-Ausschuss Nord- und Ostsee (BLANO) v. 30.3.2016, Stand 19.4.2020, abrufbar unter https:// www.meeresschutz.info/berichte-art13.html?file $=$ files $/$ meeresschutz/berichte/art13-massnahmen/MSRL_Art13_Massnahmenprogramm_Rahmentext.pdf.

73) MSRL-Maßnahmenprogramm (oben Fn. 72), S. 29-30 (Nordsee) und S. 67-69 (Ostsee).

74) Bund/Länder-Ausschuss Nord- und Ostsee: Zustand der deutschen Nordseegewässer 2018, Aktualisierung der Anfangsbewertung nach $\$ 45 \mathrm{c}$, der Beschreibung des guten Zustands der Meeresgewässer nach $₫ 45 \mathrm{~d}$ und der Festlegung von Zielen nach $\$ 45 \mathrm{e}$ des Wasserhaushaltsgesetzes zur Umsetzung der Meeresstrategie-Rahmenrichtlinie, verabschiedet von der Bund/Länder-Arbeitsgemeinschaft Nord- und Ostsee (BLANO) v. 13.12.2018, abrufbar unter https://www.meeresschutz.info/berichte-art-810.html? file $=$ files $/$ meeresschutz $/$ berichte $/$ art $8910 /$ zyklus $18 / \mathrm{Zu}$ standsbericht_Nordsee_2018.pdf, S. 27.

75) BSH: Zweiter Runder Tisch Biofouling erfolgreich, Stand 19.4.2021, abrufbar unter https://www.deutsche-flagge.de/de/ aktuelles/nachrichten-archiv/nachrichten-2020/bsh-zweiterrunder-tisch-biofouling-erfolgreich.

76) Siehe dazu Stefan/Wolff, NuR 2018, 531.

77) Vgl. dazu allgemein Gellermann, in: Landmann/Rohmer, Umweltrecht, 92. EL Februar 2020, BNatSchG \$40a Rdnr. 2 ff.

78) Vgl. dazu Gellermann, in: Landmann/Rohmer, Umweltrecht, 92. EL Februar 2020, BNatSchG \$40a Rdnr. 3.

79) Gellermann, in: Landmann/Rohmer, Umweltrecht, 92. EL Februar 2020, BNatSchG $\$ 40$ a Rdnr. 4; Gläß, in: BeckOK UmweltR, 56. Ed. 1.10.2020, BNatSchG $\$ 40$ a Rdnr. 6.

80) Vgl. Gellermann, in: Landmann/Rohmer, Umweltrecht, 92. EL Februar 2020, BNatSchG \$40a Rdnr. 21

81) Seeaufgabengesetz (SeeAufG) v. 17.6.2016, BGB1. I S. 1489, zuletzt geändert durch Art. 337 der Verordnung v. 19.6.2020, BGBl. I S. 1328.

82) Vgl. BT-Drs. 18/11942, S. 26; Gläß, in: BeckOK UmweltR, 56. Ed. 1. 10.2020, BNatSchG \$40a Rdnr. 18. 
Ausweislich der Gesetzesbegründung dient \40a Abs. 6 BNatSchG damit insbesondere der Gewährleistung der Umsetzung des BWÜ durch das SeeAufgG und die Vorschriften des Abschnitt 4 der SeeUmwVerhV ( $\int S 18$ ff. $)^{83}$, die auf dessen Grundlage erlassen wurde. ${ }^{84}$ Somit genießen die einschlägigen seeschifffahrtsrechtlichen Bestimmungen gegenüber denen des BNatSchG Vorrang. Demgegenüber haben binnenschifffahrtsrechtliche Vorschriften keinen Anteil an diesem Vorrang. ${ }^{85}$

Die Regelung in $\$ 40$ a Abs. 6 BNatSchG kann so gelesen werden, dass $\ 40 \mathrm{a}$ Abs. $1 \mathrm{BNatSchG}$ von vornherein unanwendbar ist, was freilich bezweifelt werden darf. ${ }^{86}$ In der Tat ist die absolute Formulierung von $\$ 40 \mathrm{a}$ Abs. 6 BNatSchG immer dann problematisch, wenn - wie in Bezug auf Biofouling - keine (den Anforderungen des Naturschutzes genügende) seeschifffahrtsrechtliche Regelung existiert. Auch darf der Wortlaut der Vorschrift im Hinblick auf die Voraussetzung ,durch Seeschiffe“ nicht zu weit interpretiert werden, da sonst jeder Eintrag von invasiven gebietsfremden Arten im (auch nur mittelbaren) Zusammenhang mit dem Betrieb von Schiffen erfasst wäre. Es ist aber Sinn und Zweck von $\$ 40$ a Abs. 6 BNatSchG, die Umsetzung von international harmonisierten, den Schiffsbetrieb betreffenden . Vorschriften (und zwar im Ausgangspunkt nur des BWÜ) zu gewährleisten. Vom ursprünglichen, engen Anwendungsbereich des $\$ 40$ a Abs. 6 BNatSchG ausgehend dürften also nur solche Einträge gemeint sein, die auch wirklich durch den Normalbetrieb eines Schiffes (also etwa durch Ballastwasser oder durch das Biofouling auf dem Schiffsrumpf) verursacht werden.

Nicht erfasst sind demgegenüber nicht mit dem Normalbetrieb zusammenhängende Wartungsarbeiten wie die Unterwasserreinigung von Schiffsrümpfen (sog. in-water cleaning oder IWC), bei der ebenfalls lebensfähige Organismen freigesetzt werden können. ${ }^{87}$ Die Beurteilung der Zulässigkeit von IWC in deutschen Häfen und Gewässern richtet sich wegen der damit verbundenen Gewässerbenutzung vorrangig nach dem WHG, wobei die Vorgaben des BNatSchG ebenfalls berücksichtigt werden müssen. ${ }^{8}$ Soweit eine Unterwasserreinigung in der deutschen AWZ oder von deutschen Seeschiffen in der hohen See durchgeführt werden soll, sind die Vorgaben des Hohe-See-Einbringungsgesetzes (HSEG) zu beachten. ${ }^{89}$

\subsection{Seeschifffahrtsrechtliche Vorgaben (SeeAufg G und SeeUmwVerhV)}

Zentraler Standort für umweltbezogene Bestimmungen des Seeschifffahrtsrechts, soweit sie Verhaltenspflichten betreffen $^{90}$, ist die auf Grundlage des SeeAufgG erlassene SeeUmwVerhV. ${ }^{11}$ Sie deckt mehrere verschiedene Regelungsbereiche $a b .{ }^{92} \mathrm{~W}$ ie bereits erwähnt, ist im Kontext der Verhütung der Einführung invasiver gebietsfremder Arten auf die Vorschriften zur Umsetzung des BWÜ in Abschnitt 4 der SeeUmwVerhV (\$S 18-22) zu verweisen. Zu nennen ist insbesondere das in $₫ 18$ Abs. 1 Nr. 1 SeeUmwVerhV verankerte Verbot der Einleitung von Ballastwasser, sofern nicht die Bestimmungen des BWÜ eingehalten werden oder das BSH eine Erlaubnis erteilt hat. ${ }^{93}$ Zudem ist in den $\$ \$ 20 \mathrm{f}$. SeeUmwVerhV geregelt, dass Schiffe von BWÜ-Vertragsstaaten einen Ballastwasser-Behandlungsplan und ein Ballastwasser-Tagebuch mitführen müssen. ${ }^{94}$ Im Hinblick auf Biofouling ist der Vollständigkeit halber auch darauf hinzuweisen, dass die SeeUmwVerhV in ihrem Abschnitt 3 auch das AFS-Übereinkommen umsetzt (vgl. $\iint 1$ Abs. 2 lit. b, 16-17). Regelungen zu Biofouling Management enthält die SeeUmwVerhV bisher aber nicht. Somit stellt sich die Frage, ob auf Grundlage des SeeAufgG in seiner aktuellen Fassung Vorgaben für ein Biofouling Management in die SeeUmwVerhV aufgenommen werden könnten. Wie auch den in $\$ 1$ SeeAufgG genannten Aufgaben des Bundes zu entnehmen ist, dient das See-
AufgG der Schaffung einer staatlichen Ordnung der Seeschifffahrt insbesondere zur Gewährleistung der Sicherheit des Seeverkehrs, des Schutzes der Umwelt und der Ermöglichung einer deutschen Handelsflotte mit angemessenen Arbeitsbedingungen. ${ }^{95}$

$\mathrm{Zu}$ den dem Bund auf dem Gebiet der Seeschifffahrt ${ }^{96}$ in $\$ 1$ SeeAufgG als Verbandskompetenz zugewiesenen Aufgaben gehören nach $\$ 1 \mathrm{Nr} .16$ SeeAufgG ,Maßnahmen zur Verhütung der Verbreitung fremder Organismen durch Schiffe". Diese Vorschrift dient der Abwehr der von der Schifffahrt ausgehenden Gefahr der Einführung invasiver gebietsfremder Arten und ist damit als schifffahrtspolizeiliche Regelung einzuordnen. ${ }^{97}$ Besonders genannte Maßnahmen sind die „Prüfung, Zulassung und Überwachung von Anlagen zur Behandlung von Ballastwasser und Sedimenten" sowie die im Hinblick auf die Ballastwasserbehandlung ,erforderlichen vorbereitenden Maßnahmen und internationalen Zulassungsverfahren“. In der Tat ist die Umsetzung des BWÜ der wichtigste und - soweit ersichtlich - bisher einzige Anwendungsfall des $\$ 1$ Nr. 16 SeeAufgG, was auch aus der Gesetzeshistorie hervorgeht. ${ }^{98}$ Zugleich wird aber aus dem Wortlaut (,einschließlich“) deutlich, dass es sich mit dem ausdrücklichen Verweis auf das Ballastwasser nicht um eine abschließende Regelung handelt. Auch wenn hierauf in der Gesetzesbegründung nicht eingegangen wird, ${ }^{99}$ fällt die Einführung von inva-

83) See-Umweltverhaltensverordnung v. 13. 8. 2014, BGB1. I S. 1371, zuletzt geändert durch Art. 3 der Verordnung v. 13.12.2019, BGB1. I S. 2739.

84) Vgl. BT-Drs. 18/11942, S. 26; Gläß, in: BeckOK UmweltR, 56 Ed. 1.10.2020, BNatSchG \40a Rdnr. 18.

85) Soweit die SeeUmwVerhV gem. \$3 Abs. 1 Nr. 1 SeeUmwVerhV in den auf Binnenwasserstraßen liegenden Seeschifffahrtsstraßen gilt, bleibt $₫ 40$ a Abs. 6 BNatSchG freilich anwendbar.

86) Vgl. (noch zur alten Rechtslage) Zink, Das Ballastwasser-Übereinkommen der internationalen Seeschifffahrts-Organisation von 2004: Anwendung in Nord- und Ostsee, 2016, S. 486.

87) Vgl. zur Unterwasserreinigung etwa Woods/Floerl/Jones, Marine Pollution Bulletin 2012, 1392.

88) Eine detaillierte Untersuchung der wasserrechtlichen Zulässigkeit der Unterwasserreinigung in oberirdischen Gewässern und Küstengewässern nach dem WHG muss hier aus Platzgründen unterbleiben. Fraglich ist insbesondere, ob eine - wie auch immer technisch ausgestaltete - Unterwasserreinigung gem. \$\$ 9 Abs. 1, 8 Abs. 1 WHG erlaubnispflichtig und unter Beachtung des absoluten Verbotes des Einbringens von festen Stoffen nach $\int \S 32$ Abs. 1 und 45 Abs. 1 S. 1 WHG erlaubnisfähig ist.

89) Es stellt sich insbesondere die Frage, ob eine Unterwasserreinigung sich technisch so gestalten lässt, dass dabei nicht gegen das Verbot des Einbringens von Abfällen und sonstigen Stoffen gem. \$4 S. 1 HSEG verstoßen wird.

90) Vgl. Ehlers, in: Nomos-BR/Ehlers, SeeUmwVerhV, 1. Aufl. 2021, SeeUmwVerhV $\$ 1$ Rdnr. 2.

91) Vgl. \$1 Nr. 1 SeeUmwVerhV.

92) Vgl. \$1 Nr. 2 SeeUmwVerhV.

93) Siehe dazu ausführlich Proelß/Schatz, Rechtliche Vorgaben zum Umgang mit Schiffsabwasser: Völker-, unions- und nationalrechtliche Anforderungen an Einleitungen von Scrubber-Abwasser, Ballastwasser und häuslichem Abwasser durch Schiffe, 2019, S. 93 ff.; Ehlers, in: Nomos-BR/Ehlers, SeeUmwVerhV, 1. Aufl. 2021, SeeUmwVerhV \$18 Rdnr. $1 \mathrm{f}$.

94) Siehe dazu Ehlers, in: Nomos-BR/Ehlers, SeeUmwVerhV, 1. Aufl. 2021, SeeUmwVerhV \$20 Rdnr. 1; Ehlers, in: NomosBR/Ehlers, SeeUmwVerhV, 1. Aufl. 2021, SeeUmwVerhV $\$ 21$ Rdnr. 1.

95) Ehlers, in: Nomos-BR/Ehlers, SeeaufgG, 4. Aufl. 2019, SeeaufgG Einleitung Rdnr. 1.

96) Vgl. zum Begriff der Seeschifffahrt Ehlers, in: Nomos-BR/Ehlers, SeeaufgG, 4. Aufl. 2019 SeeaufgG \$1 Rdnr. 2 f.

97) Ehlers, in: Nomos-BR/Ehlers, SeeaufgG, 4. Aufl. 2019, SeeaufgG $\$ 1$ Rdnr. 58.

98) BT-Drs. 16/7415, S. 18; Ehlers, in: Nomos-BR/Ehlers, SeeaufgG, 4. Aufl. 2019, SeeaufgG \$1 Rdnr. 58.

99) Vgl. BT-Drs. 16/7415, S. 18. 
siven gebietsfremden Arten durch Biofouling daher in den Anwendungsbereich von $\$ 1 \mathrm{Nr}$. 16 SeeAufgG. Für diese Aufgabe ist gem. $\ 5$ Abs. 1 S. 2 Nr. 4c SeeAufgG das BSH zuständig. ${ }^{100}$

Ermächtigungen des Bundesministeriums für Verkehr und digitale Infrastruktur (BMVI) ${ }^{101}$ zum Erlass von Rechtsverordnungen zur Umsetzung der in $\$ 1$ SeeAufgG genannten Aufgaben finden sich insbesondere in $\$ 9$ SeeAufgG. In $₫ 9$ Abs. 1 S. 1 SeeAufgG werden ausdrücklich auch Verordnungen ,zur Abwehr von Gefahren für die Meeresumwelt“ genannt. Der Begriff der ,Meeresumwelt" umfasst bei einer weiten Auslegung auch die biologische Vielfalt und damit den Schutz der Natur vor der Einführung invasiver gebietsfremder Arten. Diese Auslegung ist schon deshalb vorzugswürdig, weil die Voraussetzung „,zur Abwehr von Gefahren für die Meeresumwelt“ ebenso wie $\$ 1$ Nr. 16 SeeAufgG überhaupt erst zur Umsetzung des BWÜ eingefügt wurde. ${ }^{102}$ Auch die Existenz des $\$ 40 \mathrm{a}$ Abs. 6 BNatSchG spricht klar für diese Auslegung.

Derzeit ermächtigt $\$ 9$ Abs. 1 S. 1 Nr. 4b SeeAufgG zum Erlass von Rechtsverordnungen über , die Anforderungen an sowie die Prüfung, Zulassung und Überwachung von Anlagen zur Behandlung von Ballastwasser und Sedimenten einschließlich der dafür erforderlichen Verfahrensbestimmungen“. Diese Ermächtigung dient der Umsetzung von $\int 1$ Nr. 16 SeeAufgG im Hinblick auf die Umsetzung des BWÜ und daran anknüpfender das Ballastwasser betreffender Regelungen der IMO. ${ }^{103}$ Sie bildet also gerade nicht den offenen Wortlaut von $\$ 1$ Nr. 16 SeeAufgG nach, der alle „Maßnahmen zur Verhütung der Verbreitung fremder Organismen durch Schiffe" erfasst. Verordnungen, die das Biofouling Management regeln, können folglich nicht auf Grundlage des $\$ 9$ Abs. 1 S. 1 Nr. 4b SeeAufgG erlassen werden.

Es bleibt somit nur die Ermächtigung in $\$ 9$ Abs. 1 S. 1 Nr. 2 SeeAufgG betreffend ,das Verhalten auf Wasserflächen und in Häfen" i.S.d. des $\$ 1$ Nr. 2 und 3 SeeAufgG. Auf diese Verordnungsermächtigung können auch Regelungen gestützt werden, die das umweltgerechte Verhalten der Seeschifffahrt gewährleisten - darunter auch (gemeinsam mit anderen Ermächtigungen in $\$ 9$ ) die SeeUmwVerhV. ${ }^{104} \mathrm{Da}$ es sich bei der Fahrt in deutsche (Meeres-) Gewässer mit Bewuchs am Schiffsrumpf um ein Verhalten von Seeschiffen mit Auswirkungen auf die Umwelt handelt, dürfte es möglich sein, auf Grundlage von $\$ 9$ Abs. 1 S. 1 Nr. 2 SeeAufgG Seeschiffe etwa zum Aufstellen und Umsetzen eines Biofouling Management Plans sowie zum Führen eines Biofouling Record Books zu verpflichten. Zur Schaffung von Klarheit sollte der Gesetzgeber hier aber nachbessern und eine ausdrückliche Verordnungsermächtigung in $\int 9$ SeeAufgG schaffen, die $\$ 1$ Nr. 16 SeeAufgG nachgebildet ist.

\subsection{Regulierung von Biofouling Management in Neuseeland, Kalifornien und Australien}

Einige Staaten haben bereits Regelungen zu Biofouling Management getroffen, ohne auf die Schaffung verbindlicher Bestimmungen durch die IMO zu warten. Bekannt sind vor allem Regelungen von Neuseeland und den USA (und dort insbesondere der Bundesstaat Kalifornien). Auch Australien hat mittlerweile einen Regulierungsprozess angestoßen. Da diese Regelungen dem deutschen Gesetz- bzw. Verordnungsgeber als Orientierung dienen können, werden sie im Folgenden - in der gebotenen Kürze - skizziert.

\subsubsection{Neuseeland}

Das neuseeländische Ministry for Primary Industries hat im Jahr 2018 auf Grundlage seines Biosecurity Acts von $1993^{105}$ einen Craft Risk Management Standard Biofouling on Vessels Arriving to New Zealand (CRMS-BIOFOUL) ${ }^{106}$ erlassen, der Regelungen zu Biofouling Ma- nagement enthält. ${ }^{107}$ Der CRMS-BIOFOUL gilt für das Risiko der Einführung gebietsfremder Arten nach Neuseeland durch Biofouling, hat also - wie schon seine Rechtsgrundlage (der Biosecurity Act) - eine naturschutzrechtliche Stoßrichtung. ${ }^{108}$ Er findet auf Schiffe Anwendung, die in Neuseeland ankern, anlegen oder an Land gebracht werden sollen und von außerhalb der Hoheitsgewässer Neuseelands kommen, nicht aber auf Schiffe, die nicht in die Hoheitsgewässer eines anderen Landes eingefahren sind, seit sie zuletzt als vorschriftsgemäß eingestuft wurden. ${ }^{109}$

Nach dem CRMS-BIOFOUL müssen Schiffe mit einem „,sauberen Rumpf“ (,clean hull“) in Neuseeland ankommen. ${ }^{110}$ Ein Schiffsrumpf gilt als sauber, wenn kein Biofouling mit lebenden Organismen vorhanden ist, das über die in Anlage 2 des CRMS-BIOFOUL genannten Schwellenwerte hinausgeht. ${ }^{111}$ Hierfür müssen geeignete Biofouling Management Maßnahmen in Form einer Reinigung (weniger als 30 Tage vor der Ankunft in Neuseeland oder innerhalb von 24 Stunden nach der Ankunft), kontinuierlicher Instandhaltungsmaßnahmen auf Basis von ,,best practice" (also die IMO Biofouling-Richtlinien, die ausdrücklich vom CRMS-BIOFOUL integriert werden ${ }^{112}$ ) und der Anwendung alternativer genehmigter Verfahren (Zulassung nach einem neuseeländischen Biosicherheitsverfahren) getroffen werden. ${ }^{113}$

Vor Ankunft müssen den neuseeländischen Behörden folgende Informationen mitgeteilt werden: geplante Aufenthaltsdauer und -orte in Neuseeland; längere vorangegangene stationäre Aufenthalte an anderen Orten; Vorkehrungen für die Reinigung des Schiffes (falls Reinigung nach Ankunft); welche Maßnahmen zur Erfüllung der Normen angewandt werden bzw. ob der Verantwortliche sich für einen anderen durch die neuseeländischen Behörden genehmigten Craft Risk Management Plan entschieden hat. ${ }^{114}$ Darüber hinaus muss das Schiff auf Anfrage der Behörden weitere Informationen bereitstellen: über das AFS des Schiffes; ob die IMO Biofouling-Richtlinien angewandt werden (einschließlich eines Biofouling Management Plans und eines Biofouling Record Books); ggf. das aktuellste internationale AFS-Zeugnis des Schiffes; sowie Zeitpunkt und Ergebnis der letzten Biofouling Inspektion. ${ }^{115}$

100) Vgl. Ehlers, in: Nomos-BR/Ehlers, SeeaufgG, 4. Aufl. 2019, SeeaufgG $\$ 5$ Rdnr. 12.

101) Vgl. Ehlers, in: Nomos-BR/Ehlers, SeeaufgG, 4. Aufl. 2019, Seeaufg $\mathrm{S} \$ 9 \mathrm{R} d n r .29$.

102) Vgl. BT-Drs. 16/7415, S. 21.

103) Vgl. BT-Drs. 16/7415, S. 21; Ehlers, in: Nomos-BR/Ehlers, SeeaufgG, 4. Aufl. 2019, SeeaufgG \$9 Rdnr. 20.

104) Ehlers, in: Nomos-BR/Ehlers, SeeaufgG, 4. Aufl. 2019, SeeaufgG $\$ 9$ Rdnr. 7.

105) Biosecurity Act (New Zealand) v. 26.8.1993, zuletzt geändert am 7.8.2020, Stand 19.4.2021, abrufbar unter: http:// www.legislation.govt.nz/act/public/1993/0095/latest/ DLM314623.html.

106) Craft Risk Management Standard: Biofouling on Vessels Arriving to New Zealand (New Zealand Ministry for Primary Industries) v. 15.11.2018, abrufbar unter: https://www.mpi.govt. nz/dmsdocument/11668/direct.

107) Vgl. New Zealand Ministry for Primary Industries: Biofouling Management, Stand 19.4.2021, abrufbar unter https:// www.mpi.govt.nz/import/border-clearance/ships-and-boatsborder-clearance/arrival-process-steps/biofouling/biofoulingmanagement/; siehe auch Coelho, Unilateral Port State Jurisdiction: The Quest for Universality in the Prevention, Reduction and Control of Ship-Source Pollution, 2019, S. 201.

108) Sec. 1.1 Abs. 2 CRMS-BIOFOUL.

109) Sec. 1.1 Abs. 1 CRMS-BIOFOUL.

110) Sec. 2.1 Abs. 1 CRMS-BIOFOUL.

111) Sec. 2.1 Abs. 2 CRMS-BIOFOUL.

112) Sec. 1.3 Abs. 1 lit. a CRMS-BIOFOUL.

113) Sec. 2.2 CRMS-BIOFOUL.

114) Sec. 2.3 Abs. 1 CRMS-BIOFOUL.

115) Sec. 2.3 Abs. 2 CRMS-BIOFOUL. 


\subsubsection{Kalifornien}

In den USA gelten schon seit dem 21. Juni 2012 bundesweite, von der US Coast Guard erlassene Regelungen, die Anforderungen an das Biofouling Management von Schiffen stellen. ${ }^{116}$ So müssen Anker und Ankerketten gespült werden, wenn der Anker eingeholt wird, um Organismen und Sedimente an ihren Entstehungsorten zu entfernen. ${ }^{117}$ Zudem muss regelmäßig Biofouling vom Schiffsrumpf, den Rohrleitungen und Tanks entfernt und in Übereinstimmung mit den einschlägigen Bestimmungen entsorgt werden. ${ }^{118}$ Darüberhinaus muss der Ballast Water Management Plan von Schiffen auch detaillierte Verfahren zur Behandlung von Biofouling und zum Entfernen von Sedimenten enthalten. ${ }^{119}$

Von besonderem Interesse sind die deutlich umfangreicheren Biofouling Management Regulations to Minimize the Transport of Nonindigenous Species from Vessels Arriving at Californian Ports (BM-Regulations) ${ }^{120}$, die Kalifornien im Jahr 2017 erlassen hat. ${ }^{121}$ Zweck der BM-Regulations ist es, in Kalifornien auf der Grundlage der besten verfügbaren Technologie, die wirtschaftlich erreichbar ist, zügig eine Beseitigung der Einführung gebietsfremder Arten in kalifornische Gewässer oder Gewässer, die sich auf kalifornische Gewässer auswirken können, zu erreichen. ${ }^{122}$ Sie gelten - soweit keine Ausnahme einschlägig ist - für alle Schiffe, die Ballastwasser befördern oder befördern können und die in einem kalifornischen Hafen ankommen. ${ }^{123}$

Inhaltlich schreiben die BM-Regulations vor, dass Schiffe einen Biofouling Management Plan unterhalten müssen, der an Bord mitgeführt und speziell für dieses Schiff erstellt werden muss. ${ }^{124}$ Diese Verpflichtung gilt für neu gebaute Schiffe, die ab dem 1. Januar 2018 in Dienst gestellt wurden und für vorhandene Schiffe nach der ersten regulär geplanten Wartung außerhalb des Wassers am oder nach dem 1. Januar 2018. ${ }^{125}$ Die BM-Regulations definieren detaillierte inhaltliche Anforderungen an den Biofouling Management Plan. ${ }^{126}$ Er muss mindestens auf dem Stand der letzten Reparatur des Schiffes außerhalb des Wassers sein und das AFS des Schiffes im Detail beschreiben. ${ }^{127} \mathrm{Zu}$ den Anforderungen gehört auch die Einhaltung der einschlägigen Vorgaben der IMO Biofouling-Richtlinien, die ausdrücklich in die BM-Regulations integriert werden. ${ }^{128}$ Auch ein Biofouling Record Book muss geführt werden und seinerseits die Vorgaben der IMO Biofouling-Richtlinien einhalten. ${ }^{129}$ Mindestens 24 Stunden vor der ersten Ankunft eines Schiffes im Kalenderjahr muss ein Formular (Marine Invasive Species Program Annual Vessel Reporting Form) mit den relevanten Informationen schriftlich oder elektronisch bei den kalifornischen Behörden eingereicht werden. ${ }^{130}$ Für Schiffe, die seit ihrer letzten Reinigung oder Wartung eine längere Aufenthaltsdauer hatten, gelten besondere Vorschriften, die unter anderem die Beseitigung des Biofoulings verlangen. ${ }^{131}$ Zudem enthalten die BM-Regulations Vorgaben für die Durchführung der Unterwasserreinigung von Schiffen in kalifornischen Gewässern, die in Deutschland allerdings inhaltlich eher dem Wasserrecht zugerechnet werden würden. ${ }^{132}$

\subsubsection{Australien}

In Australien läuft derzeit unter Einbeziehung von interessierten Parteien ein Prozess zur Ausarbeitung verbindlicher Regeln für ein Biofouling Management. ${ }^{133}$ Bisher hat Australien zur Bekämpfung der Risiken der Einführung invasiver gebietsfremder Arten durch Biofouling an Seeschiffen auf eine Mischung aus freiwilligen „Best Practices" der Schiffsbetreiber (auf Grundlage unverbindlicher staatlicher Empfehlungen und der IMO Biofouling-Richtlinien) sowie auf im Einzelfall getroffene Maßnahmen auf Grundlage naturschutzrechtlicher Ermächtigungsgrundlagen (insbesondere des Biosecurity $\mathrm{Act}^{134}$ ) gesetzt. ${ }^{135}$ Auch in Australien wird das Thema Biofouling also grundsätzlich aus naturschutzrechtlicher Perspektive betrachtet und auch dort könnte in Zukunft eine verbindliche gesetzliche Spezialregelung eingeführt werden. Aus deutscher Sicht dürfte besonders der derzeit stattfindende Prozess der Ausarbeitung samt der in diesem Rahmen erarbeiteten Dokumente zur Konsultation interessierter Parteien interessant sein.

\section{Schlussbetrachtung}

Auf der Ebene des (See-) Völkerrechts besteht unter anderem eine allgemein gehaltene Verpflichtung aus Art. 196 Abs. 1 SRÜ, Maßnahmen zu treffen, die die Meeresumwelt vor der Einführung invasiver gebietsfremder Arten schützen. Das wichtigste globale Instrument sind aber die IMO Biofouling-Richtlinien, die detaillierte - aber nicht verbindliche - Empfehlungen für Biofouling Management enthalten. Bis zur Schaffung eines verbindlichen völkerrechtlichen Vertrags auf Ebene der IMO bleibt es also Sache einzelner Staaten oder regionaler Staatengruppen, das Biofouling Management von Seeschiffen zu regeln. Die für Deutschland wichtigste regionale Initiative findet sich im Rahmen von HELCOM, wo auch bereits ein Vorschlag für eine Biofouling Management Roadmap für die Ostsee vorhanden ist. Dabei handelt es sich zwar nicht um einen Vorschlag für konkrete verbindliche Regelungen, aber um wichtige Informationen und Empfehlungen, die bei der Schaffung regionaler und nationaler Regelungen eine Rolle spielen können.

Auf der Ebene des Unionsrechts bildet die IAVO ein allgemeines Regime zur Bekämpfung der Einführung invasiver gebietsfremder Arten, das allerdings keine spezifischen

116) 33 CFR Section 151.2050: Additional Requirements - Nonindigenous Species Reduction Practices (United States) v. 2012, Stand 19.4.2021, abrufbar unter https://www.govregs.com/ regulations/expand/title33_chapterI_part151_subpartD_section151.2050\#title33_chapterI_part151_subpartD_section 151 . 2050.

117) Vgl. 33 CFR Sec. 151.2050 lit. e.

118) Vgl. 33 CFR Sec. 151.2050 lit. f

119) Vgl. 33 CFR Sec. 151.2050 lit. g Abs. 3.

120) Biofouling Management Regulations to Minimize the Transfer of Nonindigenous Species from Vessels Arriving at California Ports (California) v. 20.4.2017, Stand 19.4.2021, abrufbar unter https://www.slc.ca.gov/laws-regulations/current-regulations/.

121) Siehe dazu Coelho, Unilateral Port State Jurisdiction: The Quest for Universality in the Prevention, Reduction and Control of Ship-Source Pollution, 2019, S. $199 \mathrm{f}$.

122) Vgl. \$2298.1. lit. a BM-Regulations.

123) Vgl. \$2298.1. lit. b BM-Regulations.

124) Vgl. \$2298.3. lit. b BM-Regulations.

125) Vgl. \$2298.3. lit. a BM-Regulation.

126) Vgl. \$2298.3. lit. b BM-Regulations.

127) Vgl. \$2298.3. lit. b BM-Regulations.

128) Vgl. \$2298.3. lit. b Abs. 2 BM-Regulations.

129) Vgl. \$2298.4. BM-Regulations.

130) Vgl. \$2298.5. BM-Regulations.

131) Vgl. \$2298.7. BM-Regulations.

132) Vgl. S\$2298.6. und 2298.8. BM-Regulations.

133) Australian Government, Department of Agriculture, Water and the Environment: Consultation Regulation Impact Statement: Developing Mandatory Biofouling Requirements, Stand 19.4.2021, abrufbar unter https://www.agriculture.gov.au/ biosecurity/avm/vessels/marine-pest-biosecurity/biofouling/ consultation-reg-impact-statement.

134) Biosecurity Act (Australia) v. 16.6.2015, zuletzt geändert am 25.3.2020, Stand 19.4.2021, abrufbar unter https://www.legislation.gov.au/Details/C2020C00127.

135) Australian Government, Department of Agriculture and Water Resources, Biosecurity Animal Division: Australian Biofouling Management Requirements for International Vessel Arrivals: Consultation Regulation Impact Statement v. 1.4.2019, Stand 19.4.2021, abrufbar unter https://haveyoursay.awe.gov. $\mathrm{au} / 44531 /$ documents/101821, S. $11 \mathrm{f}$. 
Regeln für den Eintrag von solchen Organismen durch Seeschiffe enthält. Immerhin greift der deutsche Entwurf eines IAVO-Aktionsplans die Thematik Biofouling auf, wenn auch (noch) nicht im Hinblick auf die Seeschifffahrt. Daneben ist die MSRL bzw. das nationale deutsche MSRL-Maßnahmenprogramm von Bedeutung.

Im nationalen Recht fehlen in Deutschland bisher verbindliche und speziell auf Biofouling zugeschnittene hafenstaatliche Regeln. Die Vorschriften des BNatSchG zu invasiven gebietsfremden Arten bilden ein allgemeines naturschutzrechtliches Regime, das im Einzelfall auch im Kontext von Biofouling zur Anwendung kommen kann. Allerdings erfordert der Eintrag invasiver gebietsfremder Arten durch Biofouling an Seeschiffen wegen der damit verbundenen systemischen und nicht lediglich einzelfallbezogenen Herausforderungen ein eigenes, umfassendes Regelungsregime. Dies wird ausdrücklich von $\$ 40$ a Abs. 6 BNatSchG anerkannt. Beim Thema Biofouling führt der dortige Verweis auf das SeeAufG (und mittelbar die SeeUmwVerhV) allerdings noch ins Leere, da bisher keine Regelungen zu Biofouling Management auf Grundlage des SeeAufgG geschaffen wurden. Dies ist aber auf Grundlage der Aufgabenzuweisung in $\$ 1$ Nr. 16 SeeAufgG i. V.m. der Verordnungsermächtigung in $₫ 9$ Abs. 1 S. 1 Nr. 2 SeeAufgG möglich, wenn der Gesetzgeber nicht ohnehin eine spezielle Verordnungsermächtigung in $\$ 9$ SeeAufgG schaffen möchte. Da- mit stünde einer Regelung von Biofouling Management in der SeeUmwVerhV, die sich an den IMO Biofouling-Richtlinien orientiert, nichts im Wege. Wie solche Regelungen in der Praxis aussehen können, zeigt sich an den dargestellten Beispielen aus Neuseeland und Kalifornien - sowie am derzeit laufenden Regulierungsprozess in Australien.

Open Access. Dieser Artikel wird unter der Creative Commons Namensnennung 4.0 International Lizenz veröffentlicht, welche die Nutzung, Vervielfältigung, Bearbeitung, Verbreitung und Wiedergabe in jeglichem Medium und Format erlaubt, sofern Sie den/die ursprünglichen Autor(en) und die Quelle ordnungsgemäß nennen, einen Link zur Creative Commons Lizenz beifügen und angeben, ob Änderungen vorgenommen wurden.

Die in diesem Artikel enthaltenen Bilder und sonstiges Drittmaterial unterliegen ebenfalls der genannten Creative Commons Lizenz, sofern sich aus der Abbildungslegende nichts anderes ergibt. Sofern das betreffende Material nicht unter der genannten Creative Commons Lizenz steht und die betreffende Handlung nicht nach gesetzlichen Vorschriften erlaubt ist, ist für die oben aufgeführten Weiterverwendungen des Materials die Einwilligung des jeweiligen Rechteinhabers einzuholen.

Weitere Details zur Lizenz entnehmen Sie bitte der Lizenzinformation auf http://creativecommons.org/licenses/by/4.0/deed.de.

Open Access funding enabled and organized by Projekt DEAL.

\section{Kommunale Instrumente gegen Schottergärten*}

\section{Kevin Ferber}

(c) Der/die Autor(en) 2021. Dieser Artikel ist eine Open-Access-Publikation.

Der vorliegende Beitrag möchte aufzeigen, weshalb sogenannte Schottergärten eine problematische Art der Freiflächengestaltung darstellen und welche Möglichkeiten für Kommunen bestehen, um gegen sie vorgehen zu können. Dabei wird der Ansatz einer Einordnung als bauliche Anlage verfolgt, was dazu führt, dass Eigentümer von Schottergärten mittels der bauordnungsrechtlichen Beseitigungsanordnung dazu verpflichtet werden können, diese zu entfernen. Anschließend werden Möglichkeiten für die Kommunen dargestellt, eine für die Beseitigungsanordnung notwendige materielle Illegalität der Schottergärten festzustellen oder wenigstens für zukünftige Schottergärten herzustellen. Der Beitrag versucht, das Bauordnungsrecht länderübergreifend darzustellen, kann allerdings nicht auf jede Feinheit der einzelnen Bauordnungen eingehen.

\section{Einführung in die Problematik}

Die Diskussion über ein Verbot um Schottergärten hat mit dem Inkrafttreten des Gesetzes zur Änderung des Naturschutzgesetzes und des Landwirtschafts- und Landeskulturgesetzes des Landes Baden-Württemberg ${ }^{1}$ am 23.7.2020 die Ebene der Landesgesetzgebung erreicht. Mit $\$ 21$ a Naturschutzgesetz Baden-Württemberg (NatSchG BW) hat zum ersten Mal ein Bundesland eine Norm erlassen, um explizit gegen Schottergärten vorzugehen. Dies wirft zu-

\section{Kevin Ferber,}

Wissenschaftlicher Mitarbeiter

an der Professur für Öffentliches Recht (Prof. Dr. Steffen Augsberg), Justus-Liebig-Universität Gießen,

Gießen, Deutschland nächst die Frage auf, was unter Schottergärten zu verstehen ist und weshalb sie ein Problem darstellen, dem die Kommunen und darüber hinaus auch die jeweiligen Gesetzgeber begegnen müssen.

Ein Schottergarten ist eine Fläche, welche mit Schotter oder Splitt unterschiedlicher Größe sowie vereinzelter Bepflanzung gestaltet wird. ${ }^{2}$ Die Anlage einer Vielzahl von Schottergärten erfolgt, indem auf der betreffenden Fläche zunächst die oberste Bodenschicht abgetragen wird, auf die sodann ein Vlies, eine Folie oder eine Betonschicht zur Abdichtung gegen unerwünschten Bewuchs mit Unkräutern und eingetragenen Pflanzen aufgebracht wird. ${ }^{3}$ Auf diese Schicht wird dann Schotter verteilt, um so den Effekt einer ebenen, sauberen Außenfläche zu erzielen. Als gestalterisches Element werden vereinzelt robuste und trockenheitsresistente Pflanzen in die Fläche gesetzt. Bevorzugt wird diese Art der Flächengestaltung in Vorgärten eingesetzt. ${ }^{4}$

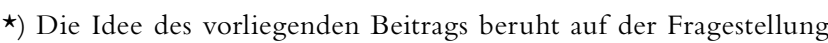
der Schwerpunktbereichshausarbeit des Verfassers vom August 2019 an der Justus-Liebig-Universität Gießen. Die zugrundeliegende Arbeit befasste sich mit der Rechtslage am Beispiel der hessischen Universitätsstadt Gießen.

1) Gesetz zur Änderung des Naturschutzgesetzes, und des Landwirtschafts- und Landeskulturgesetzes, BWGBl. 2020, 27, S. 652.

2) Krieger, Stadt+Grün 3/2016, 23; Rothenbühler, Schottergärten und Landschaft, 2017, S. 4.

3) Rothenbühler, Schottergärten und Landschaft, 2017, S. 14; VG Hannover, Urt. v. 26.11.2019 - 4 A 12592/17, juris Rdnr. 21

4) Krieger, Stadt+Grün 3/2016, 23; Rothenbühler, Schottergärten und Landschaft, 2017, S. 25. 